\title{
Prophylaxis of medication-related necrosis of the jaws in dental patients - study of 49 cases
}

\author{
Joanna Jakiel', Dagmara Jenda', Mansur Rahnama' \\ ${ }^{1}$ Chair and Department of Dental Surgery, Medical University, Lublin, Poland
}

Jakiel J, Jenda D, Rahnama M. Prophylaxis of medication-related necrosis of the jaws in dental patients - study of 49 cases. J Pre-Clin Clin Res. 2018; 12(3): 77-81. doi: 10.26444/jpccr/94396

\begin{abstract}
Introduction. Bisphosphonates and denosumab are widely used drugs in patients with bone-consuming diseases, such as osteoporosis, Paget's disease, hypercalcaemia of malignancy, bone metastases or multiple myeloma. The most important side-effect from the dental point of view is osteonecrosis of the jaw or jaw (medication-related osteonecrosis of the jaw - MRONJ). Risk factors are bone injuries, e.g. tooth extraction and other oral surgical procedures, as well as the use of mismatched restorations, bone exostoses, odontogenic inflammation, poor oral hygiene and coexisting general diseases. Treatment of MRONJ is difficult and long-lasting; therefore, special attention should be paid to the prevention of this disease. Materials and method. The study included 49 patients -29 women and 20 men. The age range was 54-77, mean age - 64. It was planned to begin bisphosphonate therapy in one patient, another was to start with denosumab therapy. The other 47 patients received BPs or denosumab. 40 of these patients received IV Bisphosphonates, 8 received oral BPs and one received denosumab.

Results. During the observation period, which varied from 1-2 years, depending on the patient's first visit, no signs of MRONJ were observed in the treated areas. There was complete mucosal healing and patients reported no pain.

Conclusions. Treatment of MRONJ is difficult and long-lasting; therefore, special attention should be paid to the prevention of this disease.
\end{abstract}

\section{Key words}

bisphosphonates, denosumab, osteonecrosis, MRONJ, prevention

\section{INTRODUCTION}

Medication-related osteonecrosis of the jaw (MRONJ) is a severe adverse drug reaction, consisting of progressive bone destruction in the maxillofacial region of patients. It is becoming more common in dental patients, presenting a growing clinical problem. For patients with malignant disease taking bisphosphonates and denosumab, the incidence of medication-related osteonecrosis of the jaw (MRONJ) is up to $15 \%$ in contrast to $0.01 \%$ in patients with osteoporosis $[1,2]$.

In 2014, the American Association of Oral and Maxillofacial Surgeons (AAOMSs) suggested changing the nomenclature from bisphosphonate-related osteonecrosis of the jaw (BRONJ) to MRONJ to accommodate the growing number of osteonecrosis cases involving the maxilla and mandible associated with other antiresorptive (denosumab) and antiangiogenic therapies [2].

Bisphosphonates (BPs) are widely used in the field of oncology, endocrinology and orthopedics. Oral BPs are used for osteoporosis and osteogenesis imperfecta $[3,4,5,6,7$, 8]. Intravenous (IV) BPs treat cancer-related conditions, including hypercalcaemia of malignancy, lytic lesions in multiple myeloma, and the effects of bone metastasis in the context of solid tumours, such as breast cancer, prostate cancer, and lung cancer. In metastatic disease of the bone, BPs prevent skeletal complications, reduce bone pain, reduce the necessity for radiotherapy or surgical treatment of the lesions, and improve the quality of life $[1,5,6,7,8]$.

Address for correspondence: Joanna Jakiel, Chair and Department of Dental Surgery, Medical University, Lublin, Poland

E-mail: joanna.m.jakiel@gmail.com

Received: 29 May 2018; Accepted: 13 August 2018
BPs are non-metabolized pyrophosphate analogues that are capable of localizing in bone and inhibiting osteoclast function $[3,6,7,8]$. These drugs act at the site of active bone remodeling by binding to hydroxyapatite, and inhibiting osteoclast development and migratory activity. Inhibiting osteoclast function leads to cell death, which decreases bone resorption without affecting bone mineralization $[3,4,8]$. These non-metabolized analogues are maintained at high concentrations in bone resorption lacunae for an extended period, allowing long-term inhibition of osteoclastic function. In addition, BPs can inhibit bone resorption and decrease bone turnover at the tissue level, as assessed by biochemical markers. In addition, bisphosphonates reduce angiogenesis by inhibition of VEGF [3,9]. BPs have also direct anti-tumoural effects, including induction of tumour cell apoptosis, inhibition of tumour cell adhesion and invasion, which reduces formation of metastases [3].

Adverse effects associated with the use of BP include pyraexia, gastrointestinal symptoms, hypocalcaemia, and renal dysfunction $[3,4,5,8]$. Bisphosphonate-related osteonecrosis of the jaws was first described in 2003 as a new complication associated with nitrogen-containing BP use $[1,4,5,6,7]$. BRONJ is diagnosed when BP administration is followed by bone exposure that does not heal within eight weeks of identification, and when patients have no history of local radiation therapy $[4,5,6,7,8,10,11]$.

Denosumab is a RANK ligand inhibitor. It is an antiresorptive medication that inhibits osteoclast function, decreases bone resorption, and increases bone density. It is used in patients affected by osteoporosis or metastatic bone diseases [2].

The reason for medication-related osteonecrosis of the jaws seems to be impaired bone turnover, and therefore 
prolonged healing of the bone due to inhibition of osteoclasts. Bisphosphonates also have a toxic affect on oral epithelium, as well as an anti-vascular effect. Consequently, local microdamage from normal mechanical loading or injury cannot be repaired which, in turn, can result in bone necrosis [4, $5,7]$. Exposed bone can be easily infected by oral biofilm, especially species of Actinomyces, Fusobacterium, Bacillus, Staphylococcus, Streptococcus viridans, Selenomonas, Neisseria, Prevotella melanogenica, as well as Candida sp. $[4,5]$.

Medication-related osteonecrosis of the jaws affects more often the mandible than the maxilla $[4,7,12]$. The risk of developing MRONJ increases after a tooth extraction or other dento-alveolar surgery, also due to the use of ill-fitting prosthetic appliances, existence of bone exostoses, oral infection, poor oral hygiene, coexisting diabetes mellitus, chemotherapy, steroid therapy, malnutrition and tobacco use $[3,4,5,6,13]$. Tooth extraction was the reason for bisphosphonate-related osteonecrosis in $34-86 \%$ of cases $[6,13,14]$. The route of administration is also important the estimated incidence of BRONJ for patients taking IV bisphosphonates ranges from $0.8-12 \%$, whereas for oral bisphosphonates it ranges from $0.01-0.04 \%$ [4, 5, 6, 10]. Most patients with BRONJ were treated with intravenous BPs, such as zolendronic acid, disodium pamidronate and sodium alendronate $[4,5,6,10,11]$. Duration of bisphosphonate exposure is positively correlated to developing BRONJ, mean time $-1.5-5$ years $[11,13]$.

\section{MATERIALS AND METHOD}

The study included 49 patients - 29 women and 20 men. Age range was $54-77$, mean age -64 . One patient was planned to start bisphosphonate therapy, one other was to start denosumab therapy. The other 47 patients received BPs or denosumab before. 40 of these patients received IV Bisphosphonates, 8 received oral BPs and one received denosumab (Tab. 1).

Patients before BPs and denosumab treatment. Of the 49 referred from the oncologists for oral assessment and dental treatment before treatment with BPs or denosumab, only 2 patients (1 male, 1 female) were referred for treatment.

The patients had scaling and root planning, and were instructed to maintain optimal oral hygiene. Extractions of necrotic roots and teeth with severe periodontitis were performed at this time. Conservative endodontic and prosthodontic treatment of teeth with good prognosis was completed. In both cases, the beginning of antiangiogenic or antiresorptive treatment was postponed as far as oral status was stable and until the surgical site has mucosalized (2-3 weeks). Chlorhexidine oral rinses were used to facilitate healing.

Inadequate dentures were modified to decrease the oral tissue pressure and to prevent sore spots. Patients were educated to report any inflammation or bone exposure. The patients were included in a periodic clinical-radiological follow-up every 3 months.

Patients receiving IV bisphosphonates, oral bisphosphonates and denosumab. 47 patients receiving bisphosphonates or denosumab, were referred for the extractions of teeth and who
Table 1.

\begin{tabular}{|c|c|c|c|c|c|}
\hline No. & Gender & $\begin{array}{l}\text { Age } \\
\text { (yrs) }\end{array}$ & $\begin{array}{l}\text { BPs/ } \\
\text { Denosumab } \\
\text { medication }\end{array}$ & $\begin{array}{l}\text { Duration of } \\
\text { medication } \\
(y r s)\end{array}$ & Type of surgery \\
\hline 1 & M & 64 & $\begin{array}{l}\text { Denosumab - } \\
\text { to be started }\end{array}$ & 0 & simple multiple extraction \\
\hline 2 & $\mathrm{~F}$ & 56 & $\begin{array}{l}\text { IV BPs - to be } \\
\text { started }\end{array}$ & 0 & simple multiple extractio \\
\hline 3 & $\mathrm{~F}$ & 62 & IV BPs & 2 & simple extraction \\
\hline 4 & $\mathrm{~F}$ & 61 & IV BPs & 3,5 & simple multiple extractions \\
\hline 5 & $\mathrm{~F}$ & 70 & IV BPs & 2,5 & simple multiple extractions \\
\hline 6 & M & 68 & IV BPs & 2,3 & simple extraction \\
\hline 7 & M & 77 & IV BPs & 4,5 & simple multiple extractions \\
\hline 8 & $\mathrm{~F}$ & 73 & oral BPs & 2 & simple extraction \\
\hline 9 & $\mathrm{~F}$ & 58 & oral BPs & 3,5 & simple extraction \\
\hline 10 & M & 66 & IV BPs & 3,2 & simple multiple extractions \\
\hline 11 & $\mathrm{~F}$ & 54 & oral BPs & 1 & simple extraction \\
\hline 12 & $\mathrm{~F}$ & 56 & oral BPs & 2 & simple extraction \\
\hline 13 & $\mathrm{~F}$ & 66 & oral BPs & 3,5 & surgical extraction \\
\hline 14 & $\mathrm{~F}$ & 71 & IV BPs & 4,5 & simple multiple extractions \\
\hline 15 & $\mathrm{~F}$ & 68 & IV BPs & 5,5 & simple multiple extractions \\
\hline 16 & $\mathrm{~F}$ & 69 & IV BPs & 4 & simple extraction \\
\hline 17 & M & 69 & IV BPs & 4,5 & simple multiple extractions \\
\hline 18 & M & 64 & IV BPs & 2,5 & simple multiple extractions \\
\hline 19 & $M$ & 64 & IV BPs & 2 & simple multiple extractions \\
\hline 20 & $M$ & 71 & IV BPs & 2,4 & simple multiple extractions \\
\hline 21 & $\mathrm{~F}$ & 64 & IV BPs & 5,5 & simple extraction \\
\hline 22 & $\mathrm{~F}$ & 75 & IV BPs & 6,6 & simple extraction \\
\hline 23 & $\mathrm{~F}$ & 72 & IV BPs & 5,8 & simple extraction \\
\hline 24 & $\mathrm{~F}$ & 62 & oral BPs & 4 & $\begin{array}{c}\text { surgical extraction, } \\
\text { oroantral communication } \\
\text { closure }\end{array}$ \\
\hline
\end{tabular}

\begin{tabular}{cccccc}
\hline 25 & M & 69 & IV BPs & 5 & simple multiple extractions \\
\hline 26 & M & 70 & IV BPs & 6,6 & simple multiple extractions \\
\hline
\end{tabular}

\begin{tabular}{|c|c|c|c|c|c|}
\hline 27 & M & 71 & IV BPs & 6,8 & simple multiple extractions \\
\hline
\end{tabular}

\begin{tabular}{llllll}
\hline 28 & M & 69 & IV BPs & 4 & simple multiple extractions \\
\hline
\end{tabular}

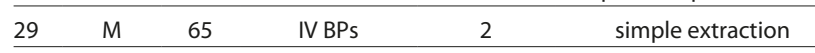

\begin{tabular}{|c|c|c|c|c|c|}
\hline 30 & M & 64 & IV BPs & 2 & simple multiple extractions \\
\hline
\end{tabular}

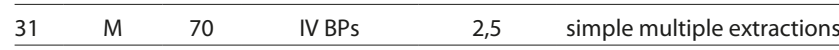

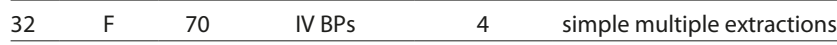

\begin{tabular}{llllll}
\hline 33 & $F$ & 77 & IV BPs & 4,5 & simple extraction \\
\hline 34 & $F$ & 67 & IV BPs & 6,7 & simple extraction
\end{tabular}

\begin{tabular}{|c|c|c|c|c|c|}
\hline 35 & $\mathrm{~F}$ & 64 & oral BPs & 4 & simple multiple extractions \\
\hline
\end{tabular}

\begin{tabular}{|c|c|c|c|c|c|}
\hline 36 & $\mathrm{~F}$ & 66 & IV BPs & 3,5 & simple multiple extractions \\
\hline
\end{tabular}

\begin{tabular}{llllll}
\hline 37 & $F$ & 66 & IV BPs & 3,5 & simple multiple extractions \\
\hline
\end{tabular}

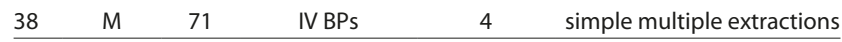

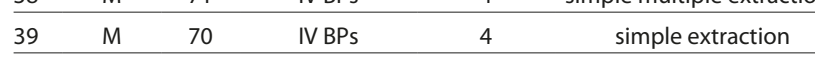

$40 \quad M \quad 60$ denosumab 2 surgical extraction,
oroantral communication closure

\begin{tabular}{cccccc}
\hline 41 & M & 62 & IV BPs & 2 & $\begin{array}{c}\text { surgical extraction, } \\
\text { oroantral communication } \\
\text { closure }\end{array}$ \\
\hline 42 & $\mathrm{~F}$ & 62 & oral BPs & 2,5 & simple extraction \\
\hline 43 & $\mathrm{~F}$ & 58 & IV BPs & 2,5 & simple extraction \\
\hline 44 & $\mathrm{~F}$ & 56 & IV BPs & 1,5 & simple multiple extractions \\
\hline 45 & $\mathrm{~F}$ & 60 & IV BPs & 2,5 & surgical extraction \\
\hline 46 & $\mathrm{~F}$ & 63 & IV BPs & 5,5 & surgical extraction \\
\hline 47 & $\mathrm{~F}$ & 68 & IV BPs & 4,5 & surgical extraction \\
\hline 48 & $\mathrm{~F}$ & 64 & IV BPs & 3,5 & simple extraction \\
\hline 49 & $\mathrm{M}$ & 68 & IV BPs & 7 & simple extraction \\
\hline
\end{tabular}


had no previous bone necrosis. The reason for extraction was untreatable pulp necrosis (29 cases), irreversible periodontitis (17 cases) and pericoronitis (1 case). 40 of these patients received IV bisphosphonates, 6 received oral BPs and one received denosumab.

Two patients reported after receiving information from the drug leaflet about possible bone necrosis. The other 40 patients were asked directly about bisphosphonate or denosumab treatment.

In 40 patients, simple tooth extractions were performed (in 18 patients single, in 22 multiple extractions), 7 were single surgical extractions. In 3 cases, immediate closure of an oro-antral communication was performed.

All patients had scaling and root planning, and were instructed to maintain optimal oral hygiene. The patients started antibiotic prophylaxis with clindamycin one day before surgery and continued for the next 6 days. Extractions were as atraumatic as possible. Afterwards, doxycycline was used until the sutures are removed. Each surgical site was sutured, and the sutures were kept in place until soft tissues healed completely (after 2-4 weeks). The patients also used chlorhexidine mouth rinses and dental adhesive paste on the site for 3-4 weeks. Dental adhesive paste contains topical anaesthetic, policanol, and the active substance, solcoseryl, a protein free standardized dialysate of culf blood. Its wound healing effect is based on activation of the intracellular metabolism and increased oxygen utilization. According to various clinical studies in the treatment of aphthae, denture pressure points and extraction wounds, the preparation accelerates the healing process by $30-50 \%$ [15].

All dentures were examined and properly fitted.

\section{RESULTS}

The study included 49 patients, 29 women and 20 men, age range $-54-77$; the means age -64 . One patients were planned to start bisphosphonate therapy, one was to start denosumab therapy. Other 47 patients received Bps or denosumab before. 40 of these patients received IV Bisphosphonates, 6 received oral BPs and one received denosumab.

During the observation period, which varied from 1-2 years depending on the patient's first visit, no signs of MRONJ were observed in the treated areas. There was complete mucosal healing and patients reported no pain.

\section{DISCUSSION}

When bisphosphonates or denosumab are recommended it is important that the prescribing physician informs patients of the risks and benefits of the drug chosen. This must include information on the risk of MRONJ and advice to attend a dental professional for a full oral assessment $[4,10,11]$. Prior to the beginning of an IV medical treatment, the patient should always be carefully evaluated by a dentist $[2,11$, 12]. The patient should be dentally fit before commencing bisphosphonate treatment $[4,11,12,13]$. If dental problems are identified, every effort should be made to have them treated before beginning bisphosphonate treatment [11, $12,16]$.

Before BPs or denosumab treatment, each patient requires oral cleansing $[11,13]$. The main goals are to remove any oral infection, pathology, or risk factors in order to obtain a stable oral health situation, preventing the necessity for invasive dental procedures in the near or intermediate future $[2,11,12,16]$ Dental hard and soft tissues should be examined for disease. Teeth currently in an acceptable condition but unlikely to be retained in the long-term need careful consideration after reflecting on the patient's dental and general health. As future exodontia is a risk factor for MRONJ, it may be sensible to consider such treatment now, particularly where long-term bisphosphonate treatment is likely $[2,11,17]$. Extraction of necrotic roots and partially impacted teeth should be performed at this time. Impacted teeth completely covered by bone without any communication with the oral cavity should be left undisturbed $[2,13,17]$. Conservative endodontic and prosthodontic therapies of teeth with good prognosis should be completed. Periodontal stabilization splints for teeth with Grades 1-2 mobility in patients with good oral hygiene and extraction in patients with poor oral hygiene are necessary $[2,13]$. If allowed by general health conditions, the beginning of antiangiogenic or anti-resorptive treatment should be deferred as far as oral status is stable or, at least, until the surgical site of extractions or other oral surgical procedures has mucosalized (2-3 weeks) $[2,8,11,13,17]$.

If a medical condition makes delay difficult or inadvisable, the most invasive procedures should be performed first, as the risk of MRONJ is associated with long-term use rather than single dosage $[4,5,17]$.

It is important to review existing dental prostheses and carefully design any that are planned, as mucosal breakdown associated with prostheses is the second most commonly identified risk factor in the development of BRONJ lesions $[4,17]$. Inadequate dentures should be modified, rebased, or replaced to decrease the oral tissue pressure and to prevent sore spots, especially along the lingual flange region or at the mandibular tori [17].

Patients should achieve a proper oral hygiene and be educated to report any grief, inflammation, or bone exposure. Patients should be included in a periodic clinical-radiological follow-up, the frequency of which is based on the medical administration, the number of risk factors, and oral health status $[4,5,11,13,18]$.

During bisphosphonates treatment it is generally recommended that high-risk procedures should be avoided, and there should be a reliance on restorative treatment, including root canal treatment and non-invasive periodontal surgery. In teeth that cannot be restored, removal of the crown and endodontic treatment of the remaining roots should be considered $[11,13,17]$. Teeth with mobility Grades 1-2 should be splinted rather than removed, and removed only in the absence of dental/periodontal lesions; extraction of teeth with mobility Grade 3 and/or endodontal-periodontal lesion. This should be completed with minimum bone injury and antibiotic treatment provided.

In the current study, preparation for oral surgical treatment, which is antibiotic prophylaxis, local antibacterial mouth rinses, suturing the wounds for a prolonged period and proper oral hygiene provided proper healing in patients taking bisphosphonates or denosumab. It is therefore suggested that atraumatic extraction, following treatment and check-ups are necessary.

An antibiotic prophylaxis for surgery procedures is necessary: Penicillin remains the first choice, in the case 
of penicillin allergy, clindamycin or a combination of quinolones-metronidazole or erythromycin-metronidazole is a valuable alternative. The authors of the current study usually use clindamycin as first choice, due to its wider antibacterial spectrum. After 6 days clindamycin, doxycycline was used until removal of the sutures. Doxycycline was chosen after surgery as it is well tolerated for prolonged antibiotic therapy [18].

Inadequate dentures should be modified, rebased, or replaced and in case of fixed prosthodontics, the biological width should be respected $[11,13,17]$.

Elective surgery and dental implants placement in patients receiving IV BPs should be avoided [16].

It is essential that a detailed oral evaluation with regular check-ups every 4-6 months for exposed bone and;early stage' MRONJ diagnosis. An orthopantomography every 6-12 months for radiographic evidence of osteosclerosis or osteolysis, widened periodontal ligament spaces, or furcation involvements, should be performed [11, 13, 17]. Good oral hygiene is essential to prevent dental infections that may require dentoalveolar surgery $[4,5,11,13,19,20]$.

Several authors have suggested a 'drug holiday' before teeth extractions or other invasive procedures. However, there is no unanimous consensus on this treatment and not enough data to support the cessation of medical treatment. If allowed by patient conditions, the oncologist should consider stopping the therapy until the healing of soft tissue occurs. As part of a preventive approach, a distinction needs to be made between IV and oral therapy in patients under medical treatment or about to initiate it [11]. However, the need for continuous dosing depends largely on the indication for the drug. While such treatment may be necessary for malignant disease, including bone metastasis, there is no evidence to suggest that a brief drug-free period would be a problem for some malignant conditions, such as hypercalcaemia and benign disease where indication for the drug is generally associated with reducing long-term risk. Any decision on drug holidays must be made in careful consultation with the prescribing physician because of the risk of adverse events if treatment is stopped [11]. The authors of the current study do not suggest a 'drug holiday' because of the extremely slow metabolism of bisphosphonates and their persistence in bones.

\section{CONCLUSIONS}

It seems that infection is closely related to MRONJ etiopathogenesis. In patients taking bisphosphonates, the site of tooth extraction favours infection because of a less inflammatory response and vascularisation of the tissues, increased bacterial adhesion to bisphosphonate-coated bone, and persistence of exposed bone to oral cavity consequent to inhibition of both bone resorption and epithelial covering [17].

Antibiotic prophylaxis together with surgical wound closure, local antiseptics and dental adhesive paste seem to be a good method for prevention of medication-related necrosis of the jaws.

Good post-operative results can be achieved by the complete mucosal sealing of the alveolar socket within the surgical extraction that prevents the bacterial contamination of the jaw [17].

Although tooth decay lesions are not directly associated with the development of MRONJ, patients must be educated regarding oral health and made aware of the consequences when caries progresses and becomes a trigger for MRONJ [20].

It is crucial to emphasize the importance of prevention of MRONJ. Indeed, elimination of all infective foci and trauma before treatment is started can almost entirely eliminate the risk of MRONJ [11].

Opportunities should be made for the users of bisphosphonates to be informed about the risks of taking these drugs. These include: revision of package inserts and drug leaflets, creation of information brochures, motivation of patients as well as doctors to improve the quality of consultations about their treatment, and perhaps even the introduction of a 'bisphosphonate passport'. Patients need to be included into a tightly integrated network of specialists in order to ensure an optimal level of information and successful therapy [12].

The treatment of MRONJ is generally difficult, and an optimal therapy strategy has still to be established. For this reason, prevention is even more important. Dental screening and adequate treatment are fundamental to reduce the risk of MRONJ in patients under anti-resorptive or antiangiogenic therapy, or before initiating the administration. $[2,7,11,12]$.

\section{REFERENCES}

1. Mücke T, Krestan CR, Mitchell DA, Kirschke JS, Wutzl A. Bisphosphonate and Medication-Related Osteonecrosis of the Jaw: A Review. Semin Musculoskelet Radiol. 2016; 20: 305-314.

2. Rosella D, Papi P, Giardino R, Cicalini E, Piccoli L, Pompa G. Medication-related osteonecrosis of the jaw: Clinical and practical guidelines. J Int Soc Prev Community Dent. 2016; 6: 97-104.

3. Van Acker HH, Anguille S, Willemen Y, Smits EL, Van Tendeloo VF. Bisphosphonates for cancer treatment: mechanisms of action and lessons from clinical trials. Pharmacol Ther 2016; 158: 24-40.

4. Patel V, McLeod NM, Rogers SN, Brennan PA. Bisphosphonate osteonecrosis of the jaw-a literature review of UK policies versus international policies on bisphosphonates, risk factors and prevention. Br J Oral Maxillofac Surg. 2011; 49: 251-257.

5. Chiapinotto Boff R, Gonc F, Salum A, Figueiredo MA, Cherubini K. Important aspects regarding the role of microorganisms in bisphosphonate-related osteonecrosis of the jaws. Arch Oral Biol. 2014; 59: 790-799.

6. American Association of Oral and Maxillofacial Surgeons Position Paper on Bisphosphonate-Related Osteonecrosis of the Jaws. J Oral Maxillofac Surg. 2007; 65: 369-376.

7. Nisi M, La Ferla F, Karapetsa D, Gennai S, Miccoli M, Baggiani A, Graziani F, Gabriele M. Risk factors influencing BRONJ staging in patients receiving intravenous bisphosphonates: a multivariate analysis. Int J Oral Maxillofac Surg. 2015; 44: 586-591.

8. Woo-Sung C, Jae-Il L, Hyun-Joong Y, Chang-Ki M, Sang-Hwa L. Medication-related osteonecrosis of the jaw: a preliminary retrospective study of 130 patients with multiple myeloma. Maxillofac Plast Reconstr Surg. 2017; 39: 1

9. De Ponte FS, Cataflamo L, Micali G, Runci M, Cutroneo G, Vermiglio G, Centfanti A, Rizzo G. Effect of bisphosphonates on the mandibular bone and gingival epithelium of rats without tooth extraction. Exp Ther Med. 2016; 11: 1678-1684.

10. Reich W, Bilkenroth U, Schubert J, Wickenhauser C, Eckert WA. Surgical treatment of bisphosphonate-associated osteonecrosis: Prognostic score and long-term results. J Craniomaxillofac Surg. 2015; 43: 1809-1822.

11. Ferlito S, Puzzo S, Palermo F, Verzì P. Treatment of bisphosphonaterelated osteonecrosis of the jaws: presentation of a protocol and an observational longitudinal study of an Italian series of cases. Br J Oral Maxillofac Surg. 2012; 50: 425-429.

12. Rupel K, Ottaviani G, Gobbo M, Contardo L, Tirelli G, Vescovi P, Di Lenarda R, Biasotto M. A systematic review of therapeutical approaches in bisphosphonates-related osteonecrosis of the jaw (BRONJ). Oral Oncol. 2014; 50: 1049-1057.

13. Bodem JF, Kargus S, Eckstein S, Saure D, Engel M, Hoffmann J, Freudlsperger C. Incidence of bisphosphonate-related osteonecrosis 
of the jaw in high-risk patients undergoing surgical tooth extraction. J Craniomaxillofac Surg. 2015; 43: 510-514.

14. Fliefel R, Tröltzsch M, Kühnisch J, Ehrenfeld M, Otto S. Treatment strategies and outcomes of bisphosphonate-related osteonecrosis of the jaw (BRONJ) with characterization of patients: a systematic review. Int J Oral Maxillofac Surg. 2015; 44: 568-585.

15. Otto S, Schreyer C, Hafner S, Mast G, Ehrenfeld M, Stürzenbaum S, Pautke C. Bisphosphonate-related osteonecrosis of the jaws: characteristics, risk factors, clinical features, localization and impact on oncological treatment. J Craniomaxillofac Surg. 2012; 40: 303-309.

16.Zadik Y, Abu-Tair J, Yarom N, Zaharia B, Elad S. The importance of thorough medical and pharmacological history before dental implant placement. Aust Dent J. 2012; 57: 388-392.
17. Fehm T, Felsenberg D, Krimmel M, Solomayer E, Wallwiener D, Hadjii P. Bisphosphonate-associated osteonecrosis of the jaw in breast cancer patients: recommendations for prevention and treatment. The Breast, 2009; 18: 213-217.

18. Story MJ, McCloud PI, Boehm G. Eur J Clin Pharmacol, 1991; 40: 419. 19. Al-Rabadi HF, Al-Warawreh A, Al-Saraireh A, Al-Qudah M. Solcoseryl Dental Adhesive Paste for prevention on alveolar osteitis. Pakistan Oral \& Dental Journal 2012; 32: 1, 23-25.

20. Bauer JS, Beck N, Kiefer J, Stockmann P, Wichmann M, Eitner S. Awareness and education of patients receiving bisphosphonates. J Craniomaxillofac Surg. 2012; 40: 277-282. 